\title{
openheart Interscallop separations of the posterior mitral valve leaflet: a solution to the 'borderline RHD' conundrum?
}

\author{
Luke David Hunter (D) , ${ }^{1}$ Mark Monaghan, ${ }^{2}$ Guy Lloyd, ${ }^{3,4,5,6}$ Carl Lombard, ${ }^{7}$ \\ Alfonso Jan Kemp Pecoraro, ${ }^{1}$ Anton Frans Doubell, ${ }^{1}$ Philipus George Herbst ${ }^{1}$
}

\begin{abstract}
- Additional material is published online only. To view, please visit the journal online (http://dx.doi.org/10.1136/ openhrt-2020-001452).
\end{abstract}

To cite: Hunter LD, Monaghan M, Lloyd G, et al. Interscallop separations of the posterior mitral valve leaflet: a solution to the 'borderline RHD' conundrum?. Open Heart 2020;7:e001452. doi:10.1136/ openhrt-2020-001452

Received 18 September 2020 Revised 5 0ctober 2020 Accepted 6 0ctober 2020
Check for updates

(C) Author(s) (or their employer(s)) 2020. Re-use permitted under CC BY-NC. No commercial re-use. See rights and permissions. Published by BMJ.

For numbered affiliations see end of article.

Correspondence to Dr Luke David Hunter; lukehunter1987@icloud.com

\section{ABSTRACT}

Objective The World Heart Federation (WHF) criteria incorporate a Doppler-based system to differentiate between 'physiological' and 'pathological' mitral regurgitation (MR) - a sole criterion sufficient for the diagnosis of WHF 'borderline' rheumatic heart disease (RHD). We have identified that interscallop separations (ISS) of the posterior mitral valve (MV) leaflet, can give rise to pathological MR in an otherwise-normal MV. We aimed to establish and compare the prevalence of ISS-related MR among South African children at high and low risk for RHD. Methods A prospective cross-sectional echocardiographic study of 759 school children (aged 1318) was performed. Cases with $M R \geq 1.5 \mathrm{~cm}$ underwent a second comprehensive study to determine the prevalence of RHD according to the WHF guideline and establish the underlying mechanism of MR.

Results 0 400 high-risk children, two met criteria for 'definite RHD' (5 per 1000 (95\% Cl 1.4 to 18.0$) ; p=0.5)$ and 11 for 'borderline RHD' (27.5 per 1000 (95\% Cl 15.4 to 48.6)). Of 359 low-risk children, 14 met criteria for borderline RHD (39 per 1000 (95\% Cl 23.4 to 64.4)). Comprehensive echocardiography identified an underlying ISS as the mechanism of isolated pathological MR in $10(83.3 \%)$ high-risk children and 11 low-risk children (78.5\%; p>0.99).

Conclusions ISS are a ubiquitous finding among South African schoolchildren from all risk profiles and are regularly identified as the underlying mechanism of WHF pathological MR in borderline RHD cases. A detailed MV assessment with an emphasis on ascertaining the underlying mechanism of dysfunction could reduce the reported numbers of screened cases misclassified as borderline RHD.

\section{INTRODUCTION}

The finding of mitral regurgitation (MR) is a critical discriminator in echocardiographic rheumatic heart disease (RHD) screening and should prompt a detailed search for morphological features of RHD. The current 2012 World Heart Federation (WHF) criteria for the diagnosis of RHD use a Doppler-based system to grade regurgitation and differentiate between the so-called 'physiological' and 'pathological' MR (online supplemental

\section{Key questions}

What is already known about this subject?

- The identification of mild mitral valve regurgitation (MR) in a child at high risk for rheumatic heart disease (RHD) is thought to be a potential marker of underlying RHD. The current screening criteria endorsed by the World Heart Federation (WHF) use Doppler-based criteria to grade the degree of MR. Screened cases with isolated mild, 'pathological' MR are classified as borderline RHD. This subcategory contributes to the majority of WHF-screen positive disease in RHD studies around the world, and ongoing longitudinal studies are underway to determine the clinical significance of a 'borderline' diagnosis.

What does this study add?

- We demonstrate that small slits in the posterior mitral valve leaflet, which we refer to as interscallop separations, are frequently the underlying cause of mild pathological MR in high-risk and low-risk South African schoolchildren. These cases are devoid of any morphological features of RHD and should not be considered as such. Using a recognised, mechanistic approach to MR, we can reproducibly identify and exclude screened cases with pathological MR, unrelated to the rheumatic process.

How might this impact on clinical practice?

- We propose that a mechanistic approach to MR in RHD screening should replace the current Dopplerbased criteria for the evaluation of MR. Using a mechanistic approach to MR will enable diseasecontrol programmes to reduce the amount of screenpositive cases misclassified as RHD significantly.

addendum A). ${ }^{1}$ The WHF criteria further categorise screened subjects with pathological MR into those with 'definite RHD' (at least two additional morphological features) and a 'borderline RHD' category in which pathological MR is sufficient as a sole criterion. Although isolated pathological MR remains non-specific as an indicator of RHD, the underlying premise has been that pathological MR identified in high-risk children 
(in the absence of an identifiable pathology), is likely to represent rheumatic involvement. ${ }^{12}$

Nevertheless, there is consensus that the borderline group represents a diverse spectrum that includes RHD, but owing to a reduction in diagnostic specificity may equally well contain cases of alternate 'pathologies', including variants considered on the 'upper limit of normal'. ${ }^{2}$ This is of concern, mainly as borderline cases with isolated WHF pathological MR constitute between $32 \%$ and $92.3 \%$ of reported cases with WHF 'screenpositive' disease. ${ }^{4-11} \mathrm{~A}$ current research priority in RHD screening is to define echocardiographic features that can better delineate the presence of true RHD and reduce the size of the borderline group. A logical approach to differentiate normal from abnormal cases in this category would be to address the WHF method of MR evaluation. The Doppler-based system, while useful in standardising the classification of mild MR, remains a non-specific assessment and does not offer additional diagnostic clarity as to the underlying aetiology of dysfunction. A mechanistic evaluation of MR built on established international guidelines and standards may allow for further differentiation within the borderline group. ${ }^{12}$

Under the auspices of the Echo in Africa programme (EIA), we have identified a common unifying mechanism of MR in a proportion of screened high-risk children with WHF pathological MR. The MR originates from slitlike separations (interscallop separation (ISS)) between the scallops of the posterior mitral valve leaflet (PMVL) and the MR is seen to move vertically down through the PMVL, rather than across the line of valvular coaptation. ISS is a ubiquitous finding throughout our high-risk cohort, and its association with MR has raised the question as to whether a possible non-rheumatic entity may be responsible.

This study aimed to establish the prevalence of ISS and ISS-related MR among a cohort of South African schoolchildren with high-risk and low-RHD risk. Furthermore, we define a mechanistic approach for MR evaluation in RHD screening that may assist in the differentiation between true RHD and the spectrum of normalcy.

\section{METHODS}

\section{Study design, setting and participants}

A prospective cross-sectional echocardiographic study was conducted. The high-risk cohort incorporated all EIAscreening data from a public, 'non-fee' paying secondary school situated in Khayelitsha, a large informal township located on the outskirts of the Cape Town Metropole. According to standardised South African measures of socioeconomic disadvantage, the Khayelitsha household income is considerably lower than the national average with a significant proportion $(>20 \%)$ of the community living below the 'poverty line'. ${ }^{13}$ The low-risk cohort comprised all screening data from a private, independent secondary school situated in the Cape Winelands. An a priori hypothesis assumed that the RHD risk profile of attending scholars (ie, risk of poverty, overcrowded households and poor access to adequate healthcare) was low. This hypothesis was supported by the school's annual tuition fee of R130 000, which put into context, is more than the total annual income in over $25 \%$ of Khayelitsha's households. ${ }^{14}$

\section{Screening procedure}

Study participants from the low-risk cohort were enrolled between March and April 2018 and the high-risk cohort in October 2018. All schoolchildren (aged 13-18) with valid consent were screened in a tailored examination room at their respective schools.

The initial screening echocardiogram was captured using a portable handheld $(\mathrm{HH})$ device (GE Vscan V.1.2, Milwaukee, USA). Screened cases with $M R \geq 1.5 \mathrm{~cm}$ qualified for a comprehensive echocardiographic study. This study was performed during the same sitting using a laptop machine (GE Vivid I). Both studies followed a standardised protocol which included a detailed analysis of the mitral valve (MV) in long-axis and short-axis including a parasternal sweep (scanning method of the MV and associated MR incorporated into our RHD screening protocol). ${ }^{15}$ All screening and comprehensive echocardiograms in the low-risk cohort were performed by the principal investigator (LDH). All screening studies in the high-risk cohort were initially captured by a team of eight British Society of Echocardiography (BSE)-accredited sonographers, under the supervision of experienced RHD screeners (LDH, GWL). All screening studies were deidentified and uploaded to an EchoPAC database for subsequent offline analysis. Standardised echocardiographic settings were used according to the WHF guideline and those described in HH-based screening studies. ${ }^{11}$

\section{Echocardiographic definitions}

Interscallop separation

ISS are defined as slit-like separations in the PMVL, identified in the parasternal short-axis view (PSSAX) while sectioning the leaflet at the tips (online supplemental clip 1). ISS are typically seen to fold open during diastole and close during systole. The PMVL has been arbitrarily divided into sections (P1, P1/P2, P2, P2/P3, P3) to assist the reviewer in approximating the location of the ISS (figure 1).

\section{Rheumatic heart disease}

RHD classification was based on the WHF diagnostic criteria for RHD (online supplemental addendum A). The criteria classify RHD as either 'definite' or 'borderline' according to a combination of WHF pathological valvular regurgitation and morphological features of RHD.

\section{Mechanistic evaluation of MR in RHD screening}

A Carpentier-style ${ }^{17}$ classification of MV regurgitation was used to identify the following mechanisms relevant to our screening population. 


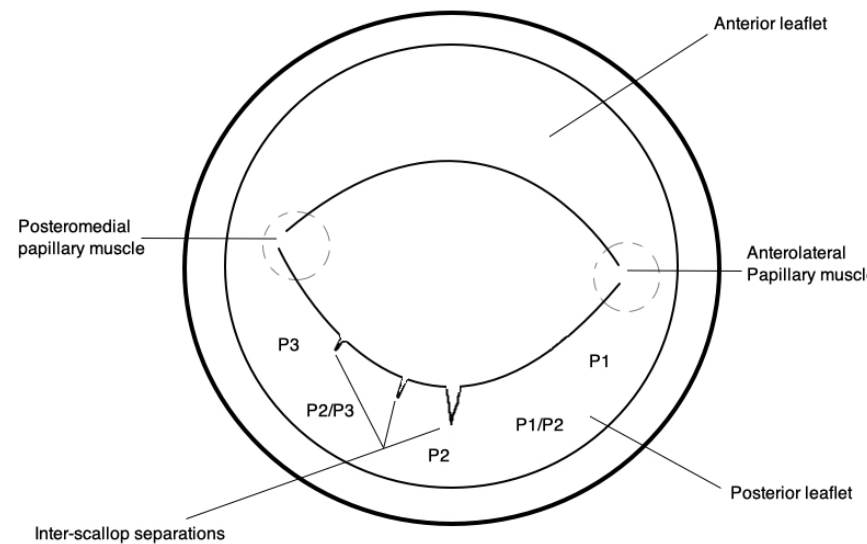

Figure 1 Echocardiographic representation of a mitral valve in the parasternal short axis view (PSSAX). The posterior leaflet has been sectioned at the tips demonstrating three examples of interscallop separation of variable location, size and shape.

\section{Normal leaflet motion}

Leaflets with normal motion were categorised into those with an MR mechanism attributable to an underlying ISS (MR originating from slit-like separations between the scallops of the PMVL) or a cleft involving the anterior mitral valve leaflet (AMVL). The origin of an ISSrelated MR jet is confirmed on an optimised PSSAX view (ensuring to section the tips of the MV leaflet). Typically, the MR jet is appreciated at, or immediately adjacent to the ISS as a fixed spot of colour or seen to be moving in a vertical up-down fashion through the PMVL rather than across the line of valvular coaptation during systole (online supplemental clip 2). The MR jet morphology must be scrutinised in an orthogonal plane (PSLAX) to exclude the possible differential of a posteriorly directed jet associated with PMVL restriction or AMVL prolapse.

\section{Excessive leaflet motion}

Leaflets with excessive motion were further categorised into cases with either mitral valve prolapse (MVP) or MVP-spectrum. MVP was diagnosed when the leaflet was seen to move beyond the annular plane $(>2 \mathrm{~mm})$ in a long axis orientation, in keeping with current consensus guidelines. ${ }^{12}$ MVP-spectrum was diagnosed in cases where some portion of the leaflet was seen to move beyond the annular plane with associated tip malcoaptation. In these cases, there was no associated PMVL restriction, nor was the valve seen to prolapse $>2 \mathrm{~mm}$ beyond the annular plane in a long axis orientation. Typically, the MR jet is seen to emanate across the line of valvular coaptation, exhibiting a broader colour Doppler jet on the optimised PSSAX view than typically seen for MR through an ISS (online supplemental clip 3 and 4).

\section{Restricted leaflet motion}

Systolic and diastolic restriction of the PMVL with resultant malcoaptation of the PMVL and AMVL during systole gives the impression of AMVL 'tip prolapse' or 'excessive leaflet motion'. These terms are synonymous and generate the so-called AMVL 'pseudoprolapse' which cannot be regarded as true prolapse, as the AMVL is seen to be in its normal position at end-systole and does not cross the annular plane. ${ }^{16}$ Pseudoprolapse of the AMVL generates the characteristic posteriorly directed jet of rheumatic MR with a similar broad Doppler jet exhibited on the optimised PSSAX view (online supplemental clips 5-7).

Restricted PMVL motion primarily during systole ('tethering') has a wide differential and includes any aetiology known to alter the geometry of the left ventricle. This category is not likely to be encountered during screening among asymptomatic children.

\section{Indeterminate}

Screened cases with MR whose underlying mechanism was not discernable were classified as 'indeterminate'.

\section{DATA ANALYSIS}

The initial screening and relevant comprehensive studies of both the high-risk and low-risk cohort were evaluated by the lead investigator ( $\mathrm{LDH})$. ISS were identified from the initial study, and only discernible cases were included in the overall count. Only the comprehensive scans of $\mathrm{HH}$ studies with positive findings $(\mathrm{MR} \geq 1.5 \mathrm{~cm})$ were reviewed and were classified according to the current WHF criteria. All screening and related comprehensive studies identified with MR were categorised according to the classification system previously described.

\section{STATISTICAL ANALYSIS}

Deidentified data were analysed using Stata (V.12, Stata Corp, Texas, USA).

Categorical variables were compared using the $\chi^{2}$ or Fisher's exact test where appropriate. A two-sided $\mathrm{p}$ value $<0.05$ was considered statistically significant. For qualitative variables, proportions along with 95\% CIs were calculated.

Cohen's kappa $(\kappa)$ statistic was used to evaluate the inter-rater agreement between the lead investigator and a second, blinded reader who was uninvolved in the initial screening (AJK). Due to the relatively small sample size, a reread of all comprehensive studies whose initial $\mathrm{HH}$ screening study had an MR jet $\geq 1.5 \mathrm{~cm}$ was performed. The lead investigator and the reader were required to note the presence of an ISS and whether the mechanism of MR was attributable to an ISS using the provided definitions. The interpretation of kappa values was based on the Landis and Koch guidelines. ${ }^{18}$ The proportion of agreement was reported as mean percentages with a $95 \%$ CI for inter-rater agreement.

\section{SAMPLE SIZE}

According to an autopsy series, ISS are present in virtually all healthy human hearts. ${ }^{19}$ The frequency of echocardiographic detection is unknown. As this is the first 
Table 1 Demographic parameters of the high-risk and lowrisk cohorts

\begin{tabular}{lcc}
\hline & $\begin{array}{l}\text { Low-risk cohort } \\
(\mathbf{n = 3 5 9 )}\end{array}$ & $\begin{array}{l}\text { High-risk cohort } \\
(\mathbf{n = 4 0 0 )}\end{array}$ \\
\cline { 2 - 2 } & $\mathbf{N}(\%)$ & $\mathbf{N}(\%)$ \\
\hline Gender (\% female) & $189(52.6)$ & $272^{*}(68)$ \\
\hline Age (mean) & 15.5 & 15.5 \\
\hline $\begin{array}{l}\text { Population group } \\
\text { Black South African }\end{array}$ & $17(4.7)$ & $400^{*}(100)$ \\
\hline $\begin{array}{l}\text { White South African } \\
\text { Mixed South African }\end{array}$ & $319^{*}(88.8)$ & $0(0)$ \\
\hline
\end{tabular}

${ }^{*} \mathrm{P}<0.001$.

comprehensive echocardiographic study of ISS, we set our anticipated frequency of detection at $50 \%$ among a population size of 1000000 . Accordingly, we determined that a sample size of 384 subjects from each cohort would accurately determine the rate of ISS between high-risk and low-risk RHD populations, with a $95 \%$ CI. The actual enrolled sample size of the low-risk cohort (359 participants) was determined to have a minimal impact on the power of the study.

\section{PATIENT AND PUBLIC INVOLVEMENT}

No patient or public involvement was used in the development of the research question or the planning of the study design.

\section{RESULTS}

\section{Cohort demographics}

A total of 759 children were enrolled for study participation. Our screening cohort comprised three population groups: black, white and mixed South African (an ethnic group of Khoisan-European-African-Malay mixed ancestry). The demographic characteristics of 400 children at high risk and 359 children at low risk are presented in table 1 . Both cohorts had a mean age of 15.5 years. The population group of the high-risk cohort was exclusively black South African $(\mathrm{p}<0.01)$ and was predominantly female $(68 \% ; \mathrm{p}<0.01)$. This is in contrast to the low-risk cohort, which was predominantly white South African $(89 \%$; $\mathrm{p}<0.01)$, with a more balanced sex distribution ( $52.6 \%$ female).

\section{WHF RHD assessment}

No low-risk child met WHF criteria for 'definite RHD' compared with two children in the high-risk cohort (prevalence, 5 per 1000 (95\% CI 1.4 to 18.0$)$; $\mathrm{p}=0.5$ ). Fourteen low-risk children (prevalence, 39 per 1000 (95\% CI 23.4 to 64.4 )) and 11 high-risk children (prevalence, 27.5 per 1000 (95\% CI 15.4 to 48.6)) met criteria for 'borderline RHD' (OR 0.69 (95\% CI 0.31 to 1.55 ); $\mathrm{p}=0.37$ ). The prevalence of RHD ('borderline' and 'definite') in the high-risk cohort was 32.5 cases per 1000 (95\% CI 19.1 to 54.8). The OR for a diagnosis of
Table 2 Frequency and location of interscallop separation(s) (ISS) detected by handheld echocardiography

\begin{tabular}{|c|c|c|}
\hline & $\begin{array}{l}\text { Low-risk cohort } \\
(\mathrm{n}=359)\end{array}$ & $\begin{array}{l}\text { High-risk cohort } \\
(n=400)\end{array}$ \\
\hline & n (\%) & $n(\%)$ \\
\hline No/indeterminate ISS* & $90(25)$ & $121(30.2)$ \\
\hline \multicolumn{3}{|l|}{ Isolated ISS† } \\
\hline P1 & $1(0.4)$ & $8 \ddagger(2.9)$ \\
\hline P2 & $40(14.8)$ & 37 (13.3) \\
\hline P3 & $42(15.6)$ & $16 \neq(5.8)$ \\
\hline P1/P2 & $6(2.2)$ & $13(4.7)$ \\
\hline P2/P3 & $136(50.6)$ & $142(51)$ \\
\hline \multicolumn{3}{|l|}{$>1$ ISS } \\
\hline Double ISS & 37 (13.8) & 55 (19.8) \\
\hline Treble ISS & $7(2.6)$ & $6(2.2)$ \\
\hline Quadruple ISS & $0(0)$ & $1(0.3)$ \\
\hline Total & $269(100)$ & $278(100)$ \\
\hline
\end{tabular}

${ }^{*}$ Calculated as a percentage of the entire cohort.

†Calculated as a percentage of cases with identifiable ISS of the posterior mitral valve leaflet. $\ddagger \mathrm{P}<0.05$.

'definite' or 'borderline RHD' in the high-risk cohort compared with the low-risk cohort was 0.82 (95\% CI 0.38 to 1.78$) ; \mathrm{p}=0.62)$.

\section{Prevalence of ISS and ISS-related MR}

A discernable ISS was identified in 278 (69.5\% (95\% CI $64.8 \%$ to $73.8 \%)$ ) cases from the high-risk cohort and in $269(74.9 \%$ (95\% CI 70.2\% to $79.1 \%)$ ) cases from the lowrisk cohort $(\mathrm{p}=0.10$; table 2$)$ A single ISS predominated in both cohorts with 216 cases $(77.7 \%$ (95\% CI $72.4 \%$ to $82.1 \%)$ ) in the high risk and 225 cases in the low-risk cohort $(62.6 \%$ (95\% CI $57.5 \%$ to $67.5 \%), \mathrm{p}<0.0001)$. Isolated ISS were most frequently identified in the P2/ P3 position, constituting the majority of ISS cases in both the high-risk ( $\mathrm{n}=142 ; 51 \%$, (95\% CI $45.2 \%$ to $56.9 \%)$ ) and low-risk cohort $(\mathrm{n}=136 ; 50.5 \% \quad(95 \%$ CI $44.6 \%$ to $56.4 \%) ; \mathrm{p}=0.93)$. MR was detected in 100 cases in the high-risk cohort (25\%; 95\% CI 21 to 29.5) and 103 cases in the low-risk cohort $(28.7 \%, 95 \%$ CI 24.26 to 33.58 ; $\mathrm{p}=0.25$; table 3 ). Overall, 547 cases were identified with ISS $(72 \%), 104$ cases of these cases had any ISS-related MR $(13.7 \%)$, but only 22 cases $(4 \%)$ demonstrated WHF pathological MR (table 3). After comprehensive echocardiography, an underlying ISS was identified as the underlying mechanism of isolated WHF pathological MR in 11 $(2.8 \%)$ high-risk children and 11 low-risk children (3\%; $\mathrm{p}=0.86$; tables 3 and 4 ). In total, ISS-related MR cases comprised 22 of the 25 WHF 'borderline RHD' cases or $88 \%$ of the total borderline group (table 3 ). There were no additional morphological or mechanistic features of RHD in these cases. 
Table 3 Amalgamated study data cross referencing mitral regurgitation (MR) prevalence, interscallop separation (ISS) and World Heart Federation (WHF) screen-positive disease

\begin{tabular}{|c|c|c|c|}
\hline & $\begin{array}{l}\text { Low-risk cohort } \\
(n=359)\end{array}$ & $\begin{array}{l}\text { High-risk cohort } \\
(n=400)\end{array}$ & $\begin{array}{l}\text { Total }(n / \%)^{*} \\
(n=759)\end{array}$ \\
\hline All ISS cases(n/\%)† & $269(74.9)$ & $278(69.5)$ & $547(72)$ \\
\hline ISS-related MR (n/\%)† & $55(15.3)$ & $49(12.3)$ & $104(13.7)$ \\
\hline ISS-related 'pathological' MR (n/\%)† & $11(3)$ & $11(2.8)$ & $22(2.9)$ \\
\hline All cases with any MR (n/\%)† & $103(28.7)$ & $100(25)$ & $203(26.7)$ \\
\hline MR screening $\geq 1.5 \mathrm{~cm}(\mathrm{n} / \%) \dagger$ & $19(5.3)$ & $23(5.8)$ & $42(5.5)$ \\
\hline WHF pathological MR (n/\%)† & $14(3.9)$ & $13(0.25)$ & $27(3.6)$ \\
\hline WHF 'screen-positive' cases (n/\%)† & $14(3.9)$ & $13(3.3)$ & $27(3.6)$ \\
\hline WHF 'borderline RHD’ (n/\%)† & $14(3.9)$ & $11(2.8)$ & $25(3.3)$ \\
\hline WHF ‘definite RHD' (n/\%)† & $0(0)$ & $2(0.5)$ & $2(0.3)$ \\
\hline
\end{tabular}

${ }^{*}$ Calculated as a percentage of the total enrolled participants.

†Calculated as a percentage of the respective cohort.

$\mathrm{MR}$, mitral regurgitation; RHD, rheumatic heart disease.

\section{Mechanistic evaluation of MR}

HH screening determined the underlying mechanism of MR in $56(56 \%)$ high-risk MR cases and $56(54.4 \%)$ lowrisk cases $(\mathrm{p}=0.88$; table 4$)$. Of these cases, ISS-related MR was identified in 49 high-risk children $(49.4 \%)$ and 55 $(53.3 \%)$ low-risk children $(\mathrm{p}=0.67)$. There were no cases of MVP identified in the high-risk cohort compared with a single case in the low-risk cohort (prevalence, 2.8 per $1000(95 \%$ CI 0.5 to 15.6$), p=0.47)$. There were five cases of MVP-spectrum identified in the high-risk cohort (prevalence, 12.5 per 1000 (95\% CI 5.4 to 28.9)) and none in the low-risk cohort $(\mathrm{p}=0.06)$. Pseudoprolapse of the AMVL was identified in both cases of WHF definite RHD. The MR mechanism on $\mathrm{HH}$ screening was indeterminate in 44 (44.4\%) high-risk children and $47(45.6 \%)$ low-risk children $(\mathrm{p}=0.39)$. Of the 27 WHF pathological MR cases, only three cases $(11.1 \%)$ had an indeterminate mechanism of MR (table 4).

\section{Assessment of interobserver agreement}

The agreement between readers on the presence of an ISS was substantial $(\kappa=0.60 ; 0.46-0.74)$ with a proportion of agreement of $88.9 \%$. There was almost perfect agreement between readers on whether the mechanism of MR was attributable to an ISS $(\kappa=0.90 ; 0.84-0.96)$ with a proportion of agreement of $96.3 \%$

\section{DISCUSSION}

This is the first descriptive study of the echocardiographic prevalence of ISS of the PMVL. Our findings suggest that ISS are a ubiquitous entity among South African children and are frequently identified as the underlying mechanism of WHF pathological MR in children, irrespective of RHD risk. An echocardiographic assessment that incorporates a mechanistic evaluation of MR may prevent misclassification of RHD in a large proportion of screened children.
The present study has three main findings. First, we present a novel, reproducible screening definition of ISS that is synonymous with previous accounts of PMVL 'slits', 'splits' and 'indentations'; a normal variant of the PMVL. ${ }^{13} 20-23$ ISS were a ubiquitous finding in our study and were identified in over two-thirds of participants in both screened cohorts (table 3). Furthermore, we present novel data suggesting that the PMVL appears to have characteristic patterns of ISS involvement. The majority of observed ISS were isolated and located in the medial aspect of the PMVL(P2/P3), accounting for over $50 \%$ of all isolated ISS in both cohorts (table 2).

Second, we have introduced a mechanistic evaluation to assess and define the mechanism of MR in RHD screening. In doing so, we reproducibly identified ISSrelated MR as the prominent mechanism of MR in the majority of cases, including those designated with WHF pathological MR (table 3).

It is important to remember that the majority of MR cases, even if designated WHF pathological MR, constituted clinical mild or very mild MR. From our experience, the exact mechanism of MR was more difficult to ascertain in those cases with the very mildest MR as the valve morphology and motion approximates normality to a high degree in these cases. Although only $55.2 \%$ of all MR cases could be allocated to a clear mechanistic group, this increased to $88.8 \%$ when considering only the WHF pathological MR group (table 4).

Although the rate of WHF pathological MR was low among all ISS-related MR cases (2.9\%), these cases constituted the bulk of the WHF borderline RHD group (table 3). ISS was identified as the mechanism in 11 highrisk $(2.8 \%)$, and $11(3 \%)$ low-risk children $(\mathrm{p}=0.86)$ with no additional morphological features of RHD included into the borderline group, constituting 22 out of the 25 cases $(88 \%)$ with borderline disease. For the first time, this finding of a common, non-rheumatic mechanism 


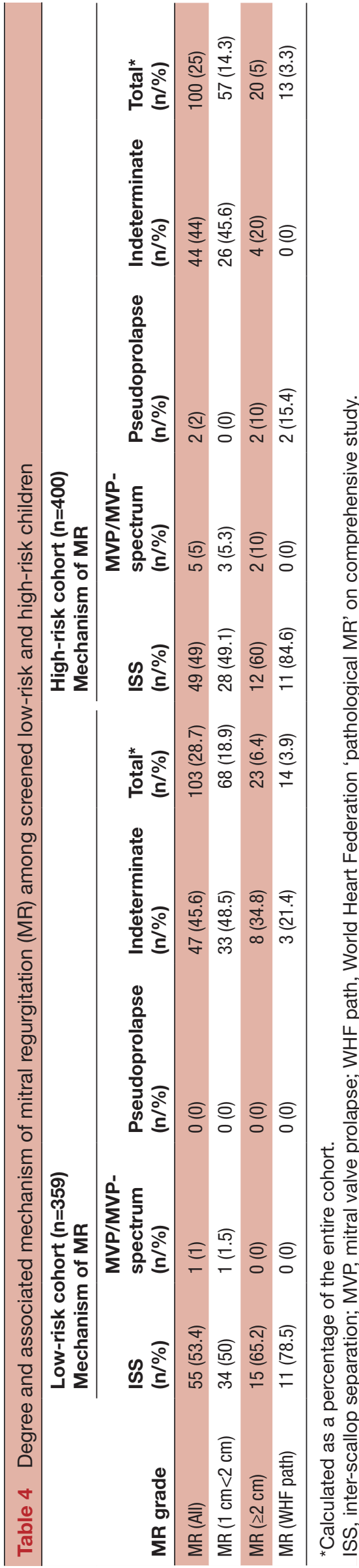

for WHF pathologic MR (ISS) challenges the dogma that WHF pathological MR necessarily points to RHD in high-risk communities. The adoption of a mechanistic evaluation of MR in RHD screening presents a critical opportunity to address the 'borderline' conundrum by significantly reducing the size of the borderline RHD group in large-scale screening studies.

The third finding of interest in this study relates to the prevalence of WHF borderline RHD identified in the low-risk cohort. While the low prevalence $(0$ cases) of WHF' definite RHD supports this particular school's a priori allocation of low-risk, we did, however, identify 14 cases of WHF pathological MR, none of which demonstrated concomitant morphological features of RHD. Our figures are appreciably higher than the 1.3\% (95\% CI $0.6 \%$ to $2.9 \%$ ) reported by Webb $e t a l^{2}$ and the $0.2 \%$ ( $95 \%$ CI $0.05 \%$ to $0.69 \%$ ) by Roberts et at ${ }^{4}$ in their respective low-risk New-Zealand and Australian cohorts. To our knowledge, this is the first published RHD screening study of low-risk children in Southern Africa and consequently have no regional data with which to compare our findings. We can only speculate as to the potential factors that may have contributed to our findings. It is possible that alternative population groups from different areas in the world may exhibit diverse MV characteristics that either predispose them to more (or less) MR. For instance, these characteristics could include variations in the number, location and size of ISS. Our EIA experience supervising volunteer BSE-accredited sonographers has highlighted an essential human factor that deserves consideration. Despite our volunteer's training, there is an associated learning curve to adequately identify mild forms of MR and in particular, capturing a complete MR CW envelope. We postulate that our unprecedented findings of borderline disease may have been in part, a function of a high quality, detailed echocardiographic study with strict adherence to the WHF criteria.

\section{Interscallop separation a cleft of the PMVL?}

The current WHF criteria stipulate that congenital causes of pathological MR (such as cleft MV) should be excluded before further analysis to avoid misidentification as RHD. ${ }^{1}$

Currently, there is no consensus on the echocardiographic definition of a PMVL cleft. Some authors define clefts by their projection into the PMVL and use an arbitrary cut-off of a depth of more than $50 \%$ of the adjacent scallop. ${ }^{21} 24$ Some require that the definition only includes clefts that extend to the annulus and are associated with some degree of regurgitation. ${ }^{20}$ Other interpretations necessitate the presence of concomitant cardiac anomalies, ${ }^{19}$ while some simply define clefts as defects located between the 'normal' interscallop position. ${ }^{21}{ }^{22} \mathrm{In}$ their autopsy series of normal hearts, Victor et al established that ISS morphology (size and number) appear to be unique to each heart. ${ }^{19}$ This finding calls into question whether instances of larger ISS 
(ie, $>50 \%$ of the PMVL) should merit an exclusive status as a cleft, having been identified as part of the normal spectrum.

While ISS has been key to the identification of the underlying mechanism of MR in a large proportion of our cases, we found the systematic assessment of ISS size using 2D echocardiography both technically challenging and imprecise. Furthermore, in our experience, there appears to be no predictable association between the anatomical size of the ISS and the degree of functional deficit. This would argue against the creation of an arbitrary definition of a PMVL' cleft' as it does not inform the screening process.

Therefore, in the screening context, it makes sense to refer to AMVL clefts as 'clefts', but an ISS should be dealt with as a normal variant of the PMVL with relevance in RHD screening. Nevertheless, given the ubiquitous nature of ISS, it is plausible that subjects with an identifiable ISS with associated MR could have concurrent true morphological features of RHD. Consequently, we would not advocate labelling a case with ISS-related MR as 'congenital', before a detailed assessment for morphological features of RHD.

\section{Limitations}

There were notable differences between the two selected cohorts of children. The population group reflected in the high-risk cohort was exclusively Black South African. This finding echoes a reality in South Africa, where the majority of Black South Africans (64.2\%) continue to live below the 'poverty line' as compared with only $1 \%$ of White South Africans. ${ }^{13}$

The sex ratio in the high-risk cohort was predominantly female, in part reflecting a documented trend of high dropout rate among males attending South African secondary schools in low socioeconomic communities. ${ }^{23}$

The size of our cohort and by implication, the relatively low prevalence of MR, limits the generalisability of our findings. Further definitive study is required to address outstanding questions that include the prevalence of ISS-related MR in larger high-risk populations, the long-term prognosis of ISS-related MR and the possibility of variability among different population groups.

The prevalence of WHF pathological ISS-related MR was not different in the high-risk and the lowrisk cohorts and supports the hypothesis that coexisting RHD is not a requirement for the development of WHF pathological MR through an ISS (table 4). However, because of the known modifying effect that ISS can have on the severity of MR in both functional and degenerative valve disease, ${ }^{24}{ }^{25}$ further study is required to evaluate the impact that coexisting RHD may have on the severity of the ISS-related MR to avoid underdiagnosis of RHD in cases with coexisting RHD and prominent ISS.

\section{CONCLUSION}

ISS of the PMVL are a ubiquitous finding among South African schoolchildren from all risk profiles and are regularly identified as the underlying mechanism of WHF pathological MR in borderline RHD cases. A detailed MV assessment with an emphasis on ascertaining the underlying mechanism of dysfunction could reduce the reported numbers of screened cases misclassified as borderline disease.

\section{Author affiliations}

'Division of Cardiology, Department of Medicine, Tygerberg Academic Hospital, Faculty of Medicine and Health Sciences, University of Stellenbosch, Cape Town, South Africa

${ }^{2}$ Cardiology, King's College London School of Medical Education, London, United Kingdom

${ }^{3}$ Barts Heart Centre, St Bartholomew's Hospital, London, United Kingdom ${ }^{4}$ Echocardiography Laboratory, Barts Heart Centre, St Bartholomew's Hospital, London, United Kingdom

${ }^{5}$ Institute of Cardiovascular Sciences, University College London, London, United Kingdom

${ }^{6}$ William Harvey Research Institute, Queen Mary University of London, London, United Kingdom

${ }^{7}$ Division of Epidemiology and Biostatistics, University of Stellenbosch, Cape Town, Western Cape, South Africa

\section{Twitter Luke David Hunter @LD_Hunter}

Acknowledgements The provision of an Edwards Lifesciences 'every heartbeat matters' grant for the Echo in Africa project is hereby acknowledged.

Contributors LDH: conceptualisation, methodology, data curation, investigation, writing-original draft preparation. MM: writing-reviewing and editing. GL: investigation, writing-reviewing and editing. $\mathrm{CL}$ : investigation and formal analysis. AJKP: investigation. AFD: supervision, writing-reviewing and editing. PGH: conceptualisation, methodology, investigation, writing-reviewing and editing, supervision.

Funding The Echo in Africa project is supported by an Edwards Lifesciences 'every heartbeat matters' grant (\#35526255).

Competing interests None declared.

Patient consent for publication Not required.

Ethics approval The study protocol conformed to the ethical guidelines of the 1975 Declaration of Helsinki as reflected in a priori approval by the University of Stellenbosch, Faculty of Health Sciences Human Research Ethics Committee (N14/04/038 \& S17/02/030) and the Western Cape Department of Education.

Provenance and peer review Not commissioned; externally peer reviewed.

Data availability statement All data related to this paper are available from the authors on reasonable request.

Open access This is an open access article distributed in accordance with the Creative Commons Attribution Non Commercial (CC BY-NC 4.0) license, which permits others to distribute, remix, adapt, build upon this work non-commercially, and license their derivative works on different terms, provided the original work is properly cited, appropriate credit is given, any changes made indicated, and the use is non-commercial. See: http://creativecommons.org/licenses/by-nc/4.0/.

ORCID iD

Luke David Hunter http://orcid.org/0000-0001-7410-1339

\section{REFERENCES}

1 Reményi B, Wilson N, Steer A, et al. World Heart Federation criteria for echocardiographic diagnosis of rheumatic heart disease--an evidence-based guideline. Nat Rev Cardiol 2012;9:297-309.

2 Webb RH, Gentles TL, Stirling JW, et al. Valvular regurgitation using portable echocardiography in a healthy student population: implications for rheumatic heart disease screening. J Am Soc Echocardiogr 2015;28:981-8. 
3 Colquhoun SM, Kado JH, Remenyi B, et al. Echocardiographic screening in a resource poor setting: borderline rheumatic heart disease could be a normal variant. Int J Cardiol 2014;173:284-9.

4 Roberts K, Maguire G, Brown A, et al. Echocardiographic screening for rheumatic heart disease in high and low risk Australian children. Circulation 2014;129:1953-61.

5 Engel ME, Haileamlak A, Zühlke $L$, et al. Prevalence of rheumatic heart disease in 4720 asymptomatic scholars from South Africa and Ethiopia. Heart 2015;101:1389-94.

6 Nascimento BR, Beaton AZ, Nunes MCP, Carmo M, et al. Echocardiographic prevalence of rheumatic heart disease in Brazilian schoolchildren: data from the PROVAR study. Int J Cardiol 2016;219:439-45.

7 Beaton A, Aliku T, Dewyer A, et al. Latent rheumatic heart disease: identifying the children at highest risk of unfavorable outcome. Circulation 2017;136:2233-44.

8 Yadeta D, Hailu A, Haileamlak A, et al. Prevalence of rheumatic heart disease among school children in Ethiopia: a multisite echocardiography-based screening. Int J Cardiol 2016;221:260-3.

9 Sims Sanyahumbi A, Sable CA, Beaton A, et al. School and community screening shows Malawi, Africa, to have a high prevalence of latent rheumatic heart disease. Congenit Heart Dis 2016;11:615-21.

10 Bertaina G, Rouchon B, Huon B, et al. Outcomes of borderline rheumatic heart disease: a prospective cohort study. Int J Cardiol 2017;228:661-5.

11 Beaton A, Lu JC, Aliku T, et al. The utility of handheld echocardiography for early rheumatic heart disease diagnosis: a field study. Eur Heart J Cardiovasc Imaging 2015;16:475-82.

12 Lancellotti P, Moura L, Pierard LA, et al. European association of echocardiography recommendations for the assessment of valvular regurgitation. Part 2: mitral and tricuspid regurgitation (native valve disease). Eur J Echocardiogr 2010;11:307-32.

13 Statistics South Africa. National poverty lines, 2018. Available: www. statssa.gov.za
14 Statistics South Africa. Census 2011- census in brief, 2011. Available: http://www.statssa.gov.za/census/census_2011/census_ products/Census_2011_Census_in_brief.pdf

15 Hunter LD, Monaghan M, Lloyd G. Prominent inter-scallop separations of the posterior leaflet of the mitral valve: an important cause of "pathological" mitral regurgitation. Echo Res Pract 2018:5:29-34.

16 Herbst P. Screening for asymptomatic rheumatic heart disease : Understanding the mechanisms key to the diagnostic criteria. $S A$ Heart 2015;12:134-44.

17 Carpentier A. Cardiac valve surgery--the "French correction". J Thorac Cardiovasc Surg 1983;86:323-37.

18 Landis JR, Koch GG. The measurement of observer agreement for categorical data. Biometrics 1977;33:159-74.

19 Victor S, Nayak VM. Definition and function of commissures, slits and scallops of the mitral valve: analysis in 100 hearts. Asia Pacific $J$ Thorac Cardiovasc Surg 1994;3:10-16.

20 Wyss CA, Enseleit F, van der Loo B, et al. Isolated cleft in the posterior mitral valve leaflet: a congenital form of mitral regurgitation. Clin Cardiol 2009;32:553-60.

21 Narang A, Addetia K, Weinert L, et al. Diagnosis of isolated cleft mitral valve using three-dimensional echocardiography. J Am Soc Echocardiogr 2018;31:1161-7.

22 Remenyi B, Gentles TL. Congenital mitral valve lesions : Correlation between morphology and imaging. Ann Pediatr Cardiol 2012;5:3.

23 Weybright EH, Caldwell LL, Xie HJ, et al. Predicting secondary school dropout among South African adolescents: a survival analysis approach. S Afr J Educ 2017;37:1-11.

24 Ring L, Rana BS, Ho SY, et al. The prevalence and impact of deep clefts in the mitral leaflets in mitral valve prolapse. Eur Heart $J$ Cardiovasc Imaging 2013;14:595-602.

25 Lai DT, Tibayan FA, Myrmel T, et al. Mechanistic insights into posterior mitral leaflet inter-scallop malcoaptation during acute ischemic mitral regurgitation. Circulation 2002;106:40-5. 